\title{
Gamma Kuvvet Fonksiyonlarının Bazı Samaryum İzotoplarının $(\gamma, n)$ ve $(\gamma, 2 n)$ Reaksiyonlarının Tesir Kesiti Hesaplamaları Üzerindeki Etkileri
}

\author{
Mert ŞEKERCI ${ }^{1 *}$ \\ ${ }^{1}$ Süleyman Demirel Üniversitesi, Fizik Bölümü, Isparta, 32260, Türkiye \\ Geliş / Received: 01/04/2020, Kabul / Accepted: 31/08/2020
}

$\ddot{O} z$

Teorik modeller ile yapılan çalışmalar, deneysel verilerin mevcut olmadığı veya deneysel çalışmaların gerçekleştirilemediği durumlarda araştırmacıların farklı veriler hakkında öngörü sahibi olmaları sağlar. Bu verilerden biri de, bir reaksiyonun meydana gelme olasıllı̆̆ olarak tanımlanabilen tesir kesiti değeridir. Ölçülebilen veya hesaplanabilen bir değer olan tesir kesitinin hesaplanmasında, çeşitli modellerin farklı etkilerinin araştırılması bu değerin doğru hesaplanabilmesi açısından son derece önemlidir. Bu bağlamda, bu çalışmada ${ }^{148,150,152,154} \mathrm{Sm}$ izotoplarının $(\gamma, \mathrm{n})$ ve $(\gamma, 2 \mathrm{n})$ reaksiyonlarında tesir kesiti hesaplamalarında farklı gama kuvvet fonksiyonlarının etkilerinin araştırılması amaçlanmıştır. Hesaplamalarda TALYS 1.9 kodu kullanılmış ve elde edilen hesaplama sonuçları literatürde mevcut olan deneysel veriler ile karşılaştırılmıştır. Ayrıca, elde edilen sonuçlar ve deneysel veriler kullanılarak göreli bağıl varyans ve ortalama ağırlıklı sapma analizleri de yapılmıştır. Bu sayede her bir reaksiyon için deneysel veriler ile en uyumlu sonuçların elde edilmesini sağlayan gama kuvvet fonksiyonu tespit edilmiştir.

Anahtar Kelimeler: Samaryum; Tesir kesiti; Gama kuvvet fonksiyonlar1; TALYS 1.9

\section{Effects of Gamma Strength Functions on Cross-Section Calculations of Some Samarium Isotopes $(\gamma, \mathbf{n})$ and $(\gamma, 2 n)$ Reactions}

\begin{abstract}
Studies with theoretical models allow researchers to have predictions about different data when experimental data are not available or experimental studies cannot be performed. One of these data is the cross-section value, which can be defined as the probability of occurrence of a reaction. In calculating the cross section, which is a value that can be measured or calculated, investigating the different effects of various models is extremely important in order to calculate this value correctly. In this context, in this study, it was aimed to investigate the effects of different gamma strength functions in the cross-section calculations in the $(\gamma, n)$ and $(\gamma, 2 \mathrm{n})$ reactions of ${ }^{148,150,152,154} \mathrm{Sm}$ isotopes. TALYS 1.9 code was used in the calculations and the results obtained were compared with the experimental data available in the literature. In addition, relative variance and mean weighted deviation analyzes were performed using the obtained results and experimental data. By this way, the gamma strength functions, which provide the most compatible results with the experimental data for each reaction, has been determined.
\end{abstract}

Keywords: Samarium; Cross-section; Gamma strength functions; TALYS 1.9

\section{Giriş}

Nükleer fizikle ilgili çalışmaların, 1896'da Henri Becquerel tarafından radyoaktivitenin keşfedilmesi ile başladığı bilinen bir gerçektir (Becquerel, 1986; Martin, 2006). Bu olayın sonrasında bu alanda gerçekleştirilen pek çok deneysel ve teorik çalışma, bilimsel ilerleme ve teknolojik gelişmeye katkı sağlamıştır. Günümüzde; endüstri ve tıp uygulamaları başta olmak üzere nükleer reaksiyonlar ve onların çıktıları pek çok alanda doğrudan veya dolaylı olarak kullanılmaktadır. $\mathrm{Bu}$ nedenle, nükleer reaksiyonlar ile reaksiyon mekanizmalarının daha iyi anlaşılması için gerçekleştirilen pek çok deneysel ve teorik çalışma bulunmaktadır. Deneysel nükleer fizik çalışmalarının daha yüksek öneme sahip olduğu gibi bir alg1 olmasına rağmen, deneysel çalışmaların gerçekleştirilemeyeceği durumlarda araştırmacılarının özel bir reaksiyona ait gözlemlenebilir parametreleri elde ederek incelemelerini sağlayacak teorik yöntem ve modeller de geliştirilmiştir. Bu modeller ile elde edilen sonuçlar; mevcut deneysel veriler ile kıyaslanarak modellerin daha tutarlı ve 
deneysel verilerin olmadığı durumlarda kabul edilebilecek sonuçlar verebilecek şekilde geliştirilmesi sağlanmıştır. Bu sayede, deneysel çalışmaların yapılamadığı durumlarda teorik çalışmaların araştırmacılara bir öngörü sağlaması amaçlanmıştır. Bir nükleer reaksiyonun gerçekleşme ihtimali olarak tanımlanabilecek tesir kesiti, incelenen reaksiyonlar ve süreçleri hakkında detaylı bilgi edinilebilmesine olanak sağladığından oldukça önemlidir. Bu bağlamda, nükleer reaksiyonlara ait gözlemlenebilir bir parametre olan ve teorik olarak da hesaplanabilen tesir kesiti ile ilgili literatürde pek çok çalışma bulunmaktadır (Kaplan vd., 2013; Yiğit vd., 2013; Kaplan vd., 2014; Kara vd., 2015; Yiğit vd., 2016; Canbula, 2017; Kavun vd., 2019; Yiğit ve Bostan, 2019). Tesir kesiti değerlerinin hesaplanmasında, reaksiyon mekanizmalarının etkilerinin incelenmesi amaciyla denge ve denge öncesi gibi nükleer reaksiyon mekanizmaları ile ilgili teorik modellerin geliştirilmiş olmasına ek olarak; seviye yoğunluğu, alfa optik model potansiyeli ve gama kuvvet fonksiyonu gibi farkl parametrelerin de incelenmesi amacıyla çeşitli teorik modeller geliştirilmiştir. Farklı modellerin, çeşitli reaksiyonlar üzerindeki etkilerinin incelendiği çalışmalar literatürde mevcuttur (Aydın vd., 2009; Aydin vd., 2015; Özdoğan, 2018; Özdoğan vd., 2018; Şekerci vd., 2019; Sarpün vd., 2019; Özdoğan vd., 2019; Şekerci vd., 2020). Benzer bir motivasyon ile, bu çalışmada gama kuvvet fonksiyonlarının bazı samaryum izotoplarına ait $(\gamma, n)$ ve $(\gamma, 2 n)$ reaksiyonlarında tesir kesiti hesaplamalarındaki etkilerinin incelenmesi amaçlanmıştır.

Samaryum, nadir toprak grubu elementlerinden biridir ve gümüşi beyaz bir metaldir. Atom numarası 62 , yoğunluğu $7,52 \mathrm{~g} / \mathrm{cm}^{3}$, en bilinen izotopu ${ }^{152} \mathrm{Sm}$ ve erime noktas $1072{ }^{\circ} \mathrm{C}$ olan samaryum, düşük toksik orana sahiptir. Optik lazerler, kızılaltı soğurucu camlar ve nükleer reaktör kontrol çubukları en yaygın kullanım alanlarıdır (Emsley, 2011; Haynes, 2014; Coursey vd., 2015). Ayrıca, ferromanyetik yapılarını yaklaşı $700 \quad{ }^{\circ} \mathrm{C}^{\prime}$ ye $\quad$ kadar koruyabildikleri ve manyetik özelliklerini kaybetmeye karşı yüksek dirence sahip oldukları bilinen samaryum-kobalt alaşımı mıknatısların küçük motorlar, kulaklıklar ve hassas güdümlü ateşli silahlarda kullanıldıkları da bilinmektedir (Chu, 2011). Medikal alanda ise, ${ }^{153} \mathrm{Sm}$ kemik kanseri tedavilerinde kullanılmaktadır (Holmes, 1992; Demir vd., 2015; Kolesnikov-Gauthier vd., 2018). Bu çalışmada samaryumun bazı izotoplarının seçilmesindeki en önemli motivasyon kaynağ 1 ise samaryumun farklı izotoplarının endüstri ve tıp uygulamalarında geniş kullanıma sahip olmalarıdır.

Çalışmanın amaçları doğrultusunda, hesaplamalarda TALYS 1.9 (Koning vd., 2017) kodu kullanılmıştır. Gama kuvvet fonksiyonları gibi teorik modeller, hesaplamalarda sofistike ve kompleks matematiksel işlemler gerektirdiği için tüm işlem adımlarının elle tamamlanmaya çalışılması hesaplamalarda hata yapılmasına ve sürecin uzamasına neden olabilir. İncelenen reaksiyon için birden çok gelen parçacık enerjisi durumunun araştırılmak istenmesi ise, her durum için hesaplamaların tekrar edilmesine ve dolayısıyla toplam işlem süresinin oldukça uzun olmasına neden olabilir. $\mathrm{Bu}$ durumdan kaçınmak ve matematiksel hata ihtimalini minimize etmek amaciyla hesaplamaların bilgisayar destekli programlar ile yapılması oldukça yaygın bir durumdur. $\mathrm{Bu}$ eksende kullanılmak üzere geliştirilmiş ALICE/ASH, EMPIRE ve TALYS gibi birçok program mevcuttur. TALYS; nükleer reaksiyonlar ve onların süreçleri ile ilgili hesaplamaların yapılabildiği, kullanıcıların çeşitli parametrelerde değişiklik yapabilmesine olanak sağlayan açık kaynak kodlu bir programdir. Programın yapısı, özellikleri ve sunduğu farklı seçenekler literatürde geniş bir kullanıma sahip olmasını sağlamıştır. Bu çalışmada, gama kuvvet fonksiyonları seçenekleri olarak TALYS kodu içeriğinde mevcut olan Kopecky-Uhl genelleştirilmiş Lorentzian (Kopecky ve Uhl, 1990), Brink-Axel Lorentzian (Brink, 1957; Axel, 1962), Hartree-Fock BCS tablolar1 
(Capote vd., 2009), Hartree-Fock-Bogolyubov tabloları (Capote vd., 2009) ve Goriely'nin hibrit model (Capote vd., 2009) kullanılmıştır. ${ }^{148,150,152,154} \mathrm{Sm}$ izotoplarının $(\gamma, \mathrm{n})$ ve $(\gamma, 2 \mathrm{n})$ reaksiyonlarında, bu gama kuvvet fonksiyonlarının etkilerinin incelenmesi amaciyla hesaplamalar tamamlanmış, elde edilen sonuçlar ise Uluslararası Deneysel Nükleer Veri Kütüphanesi (EXFOR) (Zerkin ve Pritychenko, 2018)'dan alınan mevcut deneysel verilerle karşılaştırılmıştır. Her bir reaksiyon için yapılan karşılaştırma işlemlerinin daha anlaşılabilir olması için sonuçlar hem grafikleştirilmiş, hem de bağıl varyans ve ortalama ağırlıklı sapma analizleri yapılmıştır.

\section{Materyal ve Yöntem}

Samaryum, nadir toprak grubu elementlerinden biridir ve doğal olarak oluşan yedi izotopu; doğal bolluk oranları \% 3,07 ile ${ }^{144} \mathrm{Sm}, \%$ 14,99 ile ${ }^{147} \mathrm{Sm}, \% 11,24$ ile ${ }^{148} \mathrm{Sm}, \% 13,82$ ile ${ }^{149} \mathrm{Sm}, \% 7,38$ ile ${ }^{150} \mathrm{Sm}, \% 26,75$ ile ${ }^{152} \mathrm{Sm}$ ve $\%$ 22,75 ile ${ }^{154} \mathrm{Sm}$ 'dir (Haynes, 2014; Coursey vd., 2015). Oksitleri, kalkojenidleri, halidleri, boridleri, diğer inorganik bileşenleri, organometalik bileşenleri ve bilinen otuzdan fazla izotopu ile medikalden endüstriyel uygulamalara olmak üzere çok çeşitli alanlarda kullanılmaktadırlar (Spedding vd., 1958; Jayaraman vd., 1970; Baernighausen vd., 1978; Leger vd., 1981; Evans vd., 1986). Bu çalışmada ise ${ }^{148,150,152,154} \mathrm{Sm}$ izotopları seçilerek $(\gamma, \mathrm{n})$ ve $(\gamma, 2 \mathrm{n})$ reaksiyonlarında farklı gama kuvvet fonksiyonlarının tesir kesiti hesaplamaları üzerine etkileri araştırılmıştır.

Gama kuvvet fonksiyonları, uyarılmış durumlardan foton emisyonu veya foton soğurma tesir kesiti için ortalama enerji dağılımını ifade ederler (Martin, 2012). Dolayısıyla; hem gama bozunumu hem de foton soğurmasının gama kuvvet fonksiyonları ile tanımlanması mümkündür (Özgür, 2017). Gama bozunumunun ortalama radyasyon genişliği aşağı yönlü gama kuvvet fonksiyonu ile Denklem 1'de verildiği gibi ifade edilir (Bartholomew vd., 1972).

$$
f_{X L}\left(E_{\gamma}\right)=\frac{\left\langle\Gamma_{\gamma l}\right\rangle}{\left(E_{\gamma}^{2 L+1} D_{l}\right)}
$$

Denklem 1'de, $\left\langle\Gamma_{\gamma l}\right\rangle$ ortalama 1şınımsal genişlik, $E_{\gamma}$ gama enerjisi, $D_{l}$ ise rezonans boşluğudur (Ay, 2019).

Denklem 2'de gösterilen yukarı yönlü gama kuvvet fonksiyonu (Kopecky ve Uhl 1990; Lone, 1986) ise; ortalama foton soğurma tesir kesiti $\left\langle\sigma_{X L}\left(E_{\gamma}\right)\right\rangle$ ile ilişkilidir ve tüm olası spinler üzerinden elde edilen değerlerin toplanması ile elde edilir (Özgür, 2017; Ay, 2019).

$$
f_{X L}\left(E_{\gamma}\right)=\frac{\left\langle\sigma_{X L}\left(E_{\gamma}\right)\right\rangle}{(2 L+1)\left(E_{\gamma}^{2 L-1}\right)(\pi \hbar c)^{2}}
$$

Her iki denklemde de $X$ gama kuvvet fonksiyonunun elektromanyetik karakterini, $L$ de multipolaritesini ifade etmektedir (Özgür, 2017; Ay, 2019).

Hesaplamalarda kullanılan gama kuvvet fonksiyonlarından Kopecky-Uhl genelleştirilmiş Lorentzian fonksiyonu, nötron girişli reaksiyonlarda; Brink-Axel Lorentzian fonksiyonu ise diğer tüm reaksiyonlarda TALYS tarafindan ön tanımlı model olarak seçilmektedir. Kopecky-Uhl genelleştirilmiş Lorentzian fonksiyonu Denklem 3'de gösterildiği şekilde verilebilir.

$$
\begin{aligned}
f_{E 1}\left(E_{\gamma}, T\right)=K_{E 1}\left[\frac{E_{\gamma} \tilde{\Gamma}_{E 1}\left(E_{\gamma}\right)}{\left(E_{\gamma}^{2}-E_{E 1}^{2}\right)^{2}+E_{\gamma}^{2} \tilde{\Gamma}_{E 1}\left(E_{\gamma}\right)^{2}}\right. \\
\left.+\frac{0,7 \Gamma_{E 1} 4 \pi^{2} T^{2}}{E_{E 1}^{3}}\right] \sigma_{E 1} \Gamma_{E 1}
\end{aligned}
$$

Denklem 3'deki enerji bağımlı sönümleme genişliği $\widetilde{\Gamma}_{E 1}$ ile nükleer sıcaklık $T$ ise Denklem 4 ve Denklem 5'de gösterilmiştir.

$$
\begin{gathered}
\tilde{\Gamma}_{E 1}\left(E_{\gamma}\right)=\Gamma_{E 1} \frac{E_{\gamma}^{2}+4 \pi^{2} T^{2}}{E_{E 1}^{2}} \\
T=\sqrt{\frac{E_{n}+S_{n}-\Delta-E_{\gamma}}{a\left(S_{n}\right)}}
\end{gathered}
$$

Denklem 5'de $E_{n}$ gelen nötron enerjisini, $S_{n}$ nötron ayırma enerjisini, $\Delta$ seviye yoğunluğuna 
göre eşleştirme düzeltmesini ve $a$ ise seviye deneysel tesir kesiti verisindeki hatayı ifade yoğunluğu parametresini ifade etmektedir.

etmektedir.

Brink-Axel Lorentzian fonksiyonunda ise dev dipol rezonans bölgesi standart bir Lorentzian yapı ile tanımlanmıştır. Bu duruma ait eşitlik Denklem 6 ve Denklem 7 ile gösterilmiştir.

$$
\begin{gathered}
f_{X \ell}\left(E_{\gamma}\right)=K_{X \ell} \frac{\sigma_{X \ell} E_{\gamma} \Gamma_{X \ell}^{2}}{\left(E_{\gamma}^{2}-E_{X \ell}^{2}\right)^{2}+E_{\gamma}^{2} \Gamma_{X \ell}^{2}} \\
K_{X \ell}=\frac{1}{(2 \ell+1) \pi^{2} \hbar^{2} c^{2}}
\end{gathered}
$$

$\mathrm{Bu}$ eşitliklerde $\sigma_{X \ell}, E_{X \ell}$ ve $\Gamma_{X \ell}$ dev dipol rezonansa ait şiddet, enerji ve genişlik değerleridir (Koning vd., 2017)

$\mathrm{Bu}$ çalışmada, incelenen her reaksiyon için Kopecky-Uhl genelleştirilmiş Lorentzian, Brink-Axel Lorentzian, Hartree-Fock BCS tablolar1, Hartree-Fock-Bogolyubov tablolar1 ve Goriely'nin hibrit model fonksiyonları ayrı ayrı kullanılarak hesaplamalar tamamlanmıştır. Elde edilen sonuçların, hesaplamalardaki etkilerinin incelenebilmesi için her bir reaksiyon için ilgili reaksiyona ait deneysel veriler Uluslararası Deneysel Nükleer Veri Kütüphanesi (EXFOR)'dan alınmış ve hesaplama sonuçları ile deneysel veriler görsel analiz için birlikte grafikleştirilmiştir. Buna ek olarak, sayısal bir değerlendirme yapabilmek için her bir reaksiyona ait hesaplama sonuçları ile deneysel veriler Denklem 8 (Kurenkov vd., 1999) ve Denklem 9 (Kurenkov vd., 1999) ile gösterilen eşitlikler kullanılarak göreli bağıl varyans ve ortalama ağırlıklı sapma analizleri yapılmıştır.

$$
\begin{gathered}
D=\frac{1}{N} \sum_{i=1}^{N}\left|\sigma_{i}^{\text {hes }}-\sigma_{i}^{d e n}\right| / \sigma_{i}^{d e n} \\
F=\sqrt{\left[\frac{1}{N} \sum_{i=1}^{N}\left[\left(\sigma_{i}^{\text {hes }}-\sigma_{i}^{d e n}\right) / \Delta \sigma_{i}^{d e n}\right]^{2}\right]}
\end{gathered}
$$

Denklem 8 ve Denklem 9'daki ifadelerden $N$ veri sayısın1, $\sigma_{i}^{\text {hes }}$ hesaplamalar sonucunda elde edilmiş tesir kesiti ifadesini, $\sigma_{i}^{\text {den }}$ deneysel tesir kesiti verisini ve $\Delta \sigma_{i}^{\text {den }}$ ise

\section{Bulgular}

Bu çalışmada; Kopecky-Uhl genelleştirilmiş Lorentzian, Brink-Axel Lorentzian, HartreeFock BCS tablolar, Hartree-FockBogolyubov tabloları ve Goriely'nin hibrit model gama kuvvet fonksiyonlarının ${ }^{148} \mathrm{Sm}(\gamma, \mathrm{n}){ }^{147} \mathrm{Sm}, \quad{ }^{150} \mathrm{Sm}(\gamma, \mathrm{n}){ }^{149} \mathrm{Sm}$, ${ }^{152} \mathrm{Sm}(\gamma, \mathrm{n}){ }^{151} \mathrm{Sm}, \quad{ }^{154} \mathrm{Sm}(\gamma, \mathrm{n}){ }^{153} \mathrm{Sm}$, ${ }^{148} \mathrm{Sm}(\gamma, 2 \mathrm{n}){ }^{146} \mathrm{Sm}, \quad{ }^{150} \mathrm{Sm}(\gamma, 2 \mathrm{n}){ }^{148} \mathrm{Sm}$, ${ }^{152} \mathrm{Sm}(\gamma, 2 \mathrm{n}){ }^{150} \mathrm{Sm} \quad$ ve $\quad{ }^{154} \mathrm{Sm}(\gamma, 2 \mathrm{n}){ }^{152} \mathrm{Sm}$ reaksiyonlarının tesir kesiti hesaplamalarına olan etkilerinin araştırılması amaçlanmıştır. $\mathrm{Bu}$ amaç doğrultusunda gerçekleştirilen hesaplama sonuçları; her bir reaksiyon için o reaksiyona ait literatrürde mevcut deneysel veriler ile Şekil 1-8'de karşılaştırılmıştır. Ayrıca, her bir reaksiyon için göreli bağıl varyans ve ortalama ağırlıklı sapma analizleri yapılarak sonuçlar Tablo 1 ve Tablo 2'de gösterilmiştir.

${ }^{148} \mathrm{Sm}(\gamma, n){ }^{147} \mathrm{Sm}$,
${ }^{152} \mathrm{Sm}(\gamma, n){ }^{151} \mathrm{Sm}$ reaksiyonlarına ait gama kuvvet fonksiyonları kullanılarak gerçekleştirilen tesir kesiti hesaplama sonuçları sırasıyla Şekil 1-4'de gösterilmiştir. Hesaplama sonuçlarının deneysel veriler ile karşılaştırılması amacıyla ${ }^{148} \mathrm{Sm}(\gamma, \mathrm{n}){ }^{147} \mathrm{Sm}, \quad{ }^{150} \mathrm{Sm}(\gamma, \mathrm{n}){ }^{149} \mathrm{Sm} \quad$ ve ${ }^{154} \mathrm{Sm}(\gamma, n){ }^{153} \mathrm{Sm}$ reaksiyonları literatürde mevcut olan için Filipescu vd. (2014)'ye ait deneysel veriler kullanılmıştır. ${ }^{152} \mathrm{Sm}(\gamma, \mathrm{n}){ }^{151} \mathrm{Sm}$ reaksiyonu için ise; Hara vd. (2007) ile Filipescu vd. (2014)'nin deneysel sonuçları birlikte kullanılmıştır. Elde edilen hesaplama sonuçlarının Şekil 1-4 ile gösterilen grafiksel karşılaştırmaları ile Tablo 1 ve Tablo 2 ile verilen göreli bağıl varyans ve ortalama ağırlıklı sapma analizlerine göre; ${ }^{148} \mathrm{Sm}(\gamma, \mathrm{n}){ }^{147} \mathrm{Sm}, \quad{ }^{150} \mathrm{Sm}(\gamma, \mathrm{n}){ }^{149} \mathrm{Sm} \quad$ ve ${ }^{154} \mathrm{Sm}(\gamma, \mathrm{n}){ }^{153} \mathrm{Sm}$ reaksiyonlarında deneysel veriler ile en uyumlu sonuçların gama kuvvet fonksiyonu olarak Goriely'nin hibrit modelinin kullanılması durumunda elde edildiğini 


göstermiştir. ${ }^{152} \mathrm{Sm}(\gamma, \mathrm{n}){ }^{151} \mathrm{Sm}$ reaksiyonu için sonuçları Brink-Axel gama kuvvet
ise deneysel veriler ile en uyumlu hesaplama fonksiyonunun kullanılması ile elde edilmiştir.

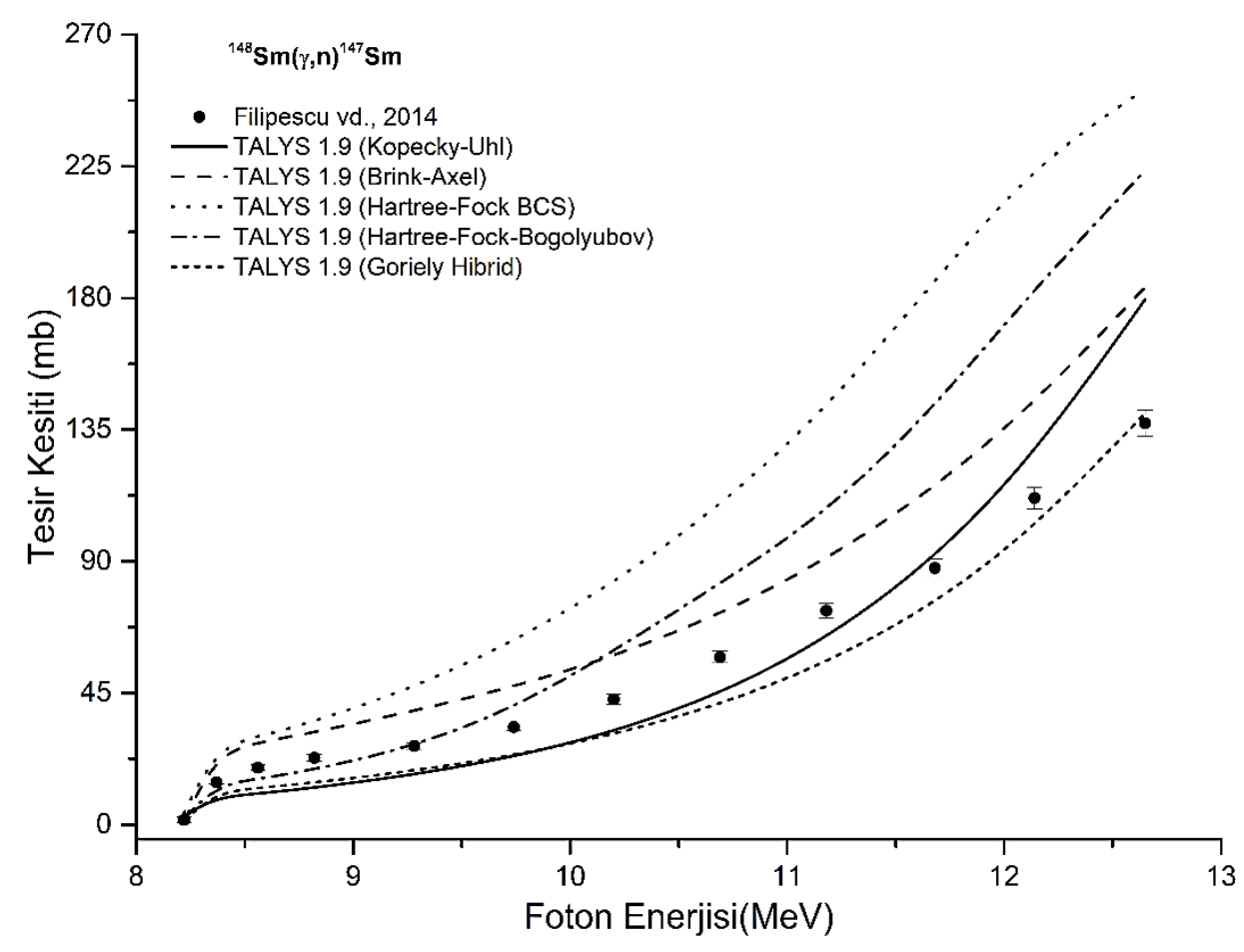

Şekil 1. ${ }^{148} \mathrm{Sm}(\gamma, \mathrm{n}){ }^{147} \mathrm{Sm}$ reaksiyonu için gama kuvvet fonksiyonları ile elde edilen teorik tesir kesiti hesaplamalarının deneysel veriler ile kıyaslanması

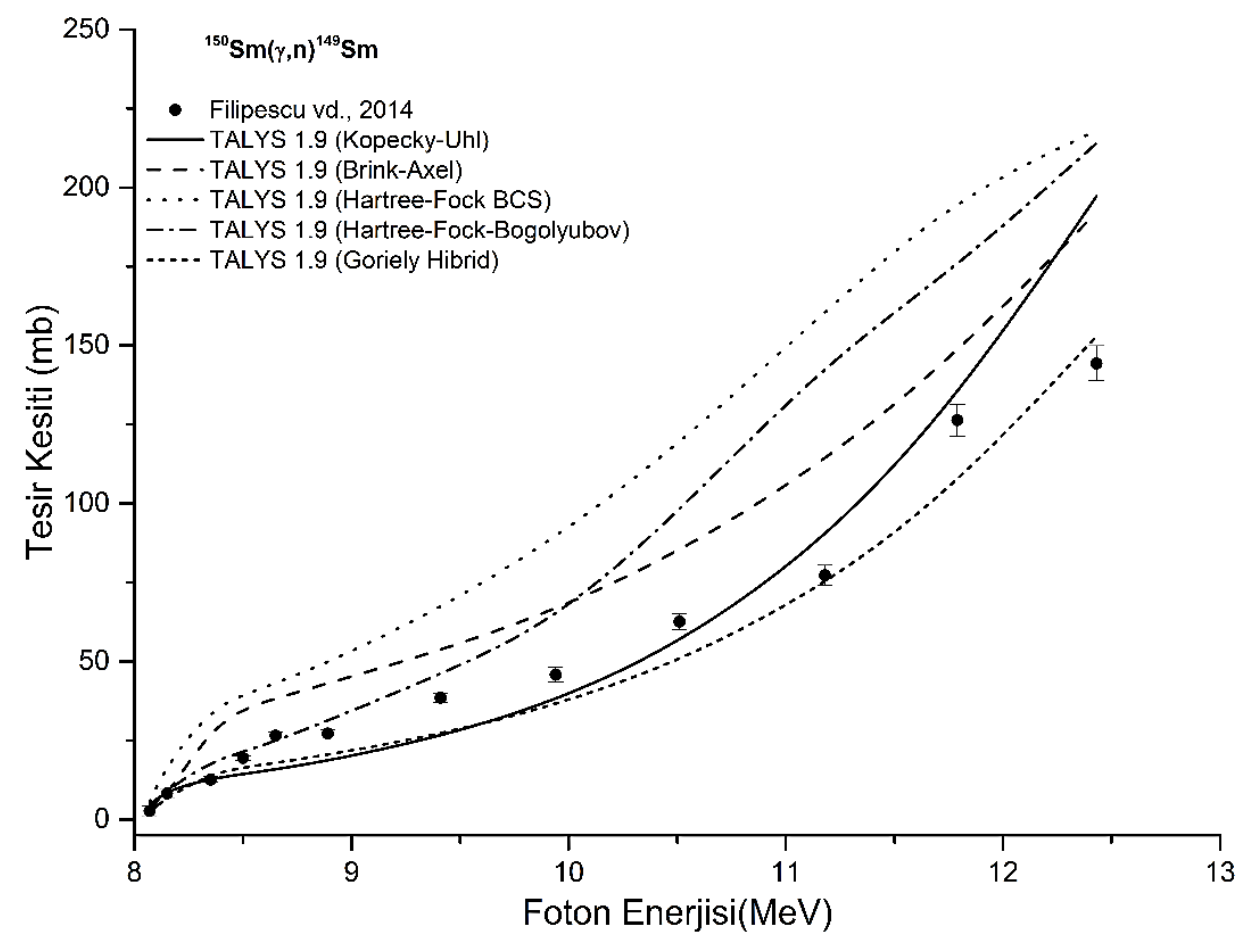

Şekil 2. ${ }^{150} \mathrm{Sm}(\gamma, \mathrm{n}){ }^{149} \mathrm{Sm}$ reaksiyonu için gama kuvvet fonksiyonları ile elde edilen teorik tesir kesiti hesaplamalarının deneysel veriler ile kıyaslanması 


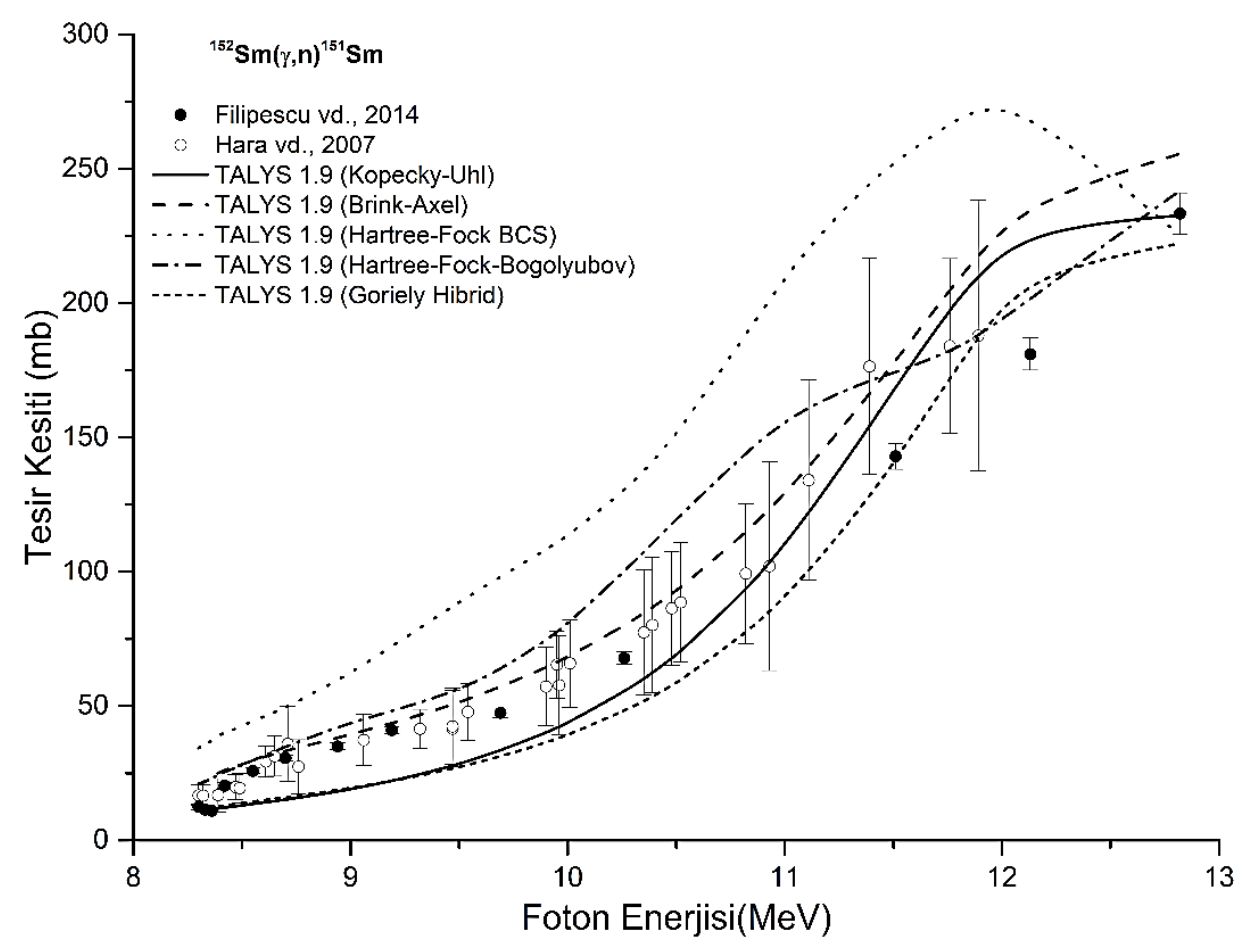

Şekil 3. ${ }^{152} \mathrm{Sm}(\gamma, \mathrm{n}){ }^{151} \mathrm{Sm}$ reaksiyonu için gama kuvvet fonksiyonları ile elde edilen teorik tesir kesiti hesaplamalarının deneysel veriler ile kıyaslanması

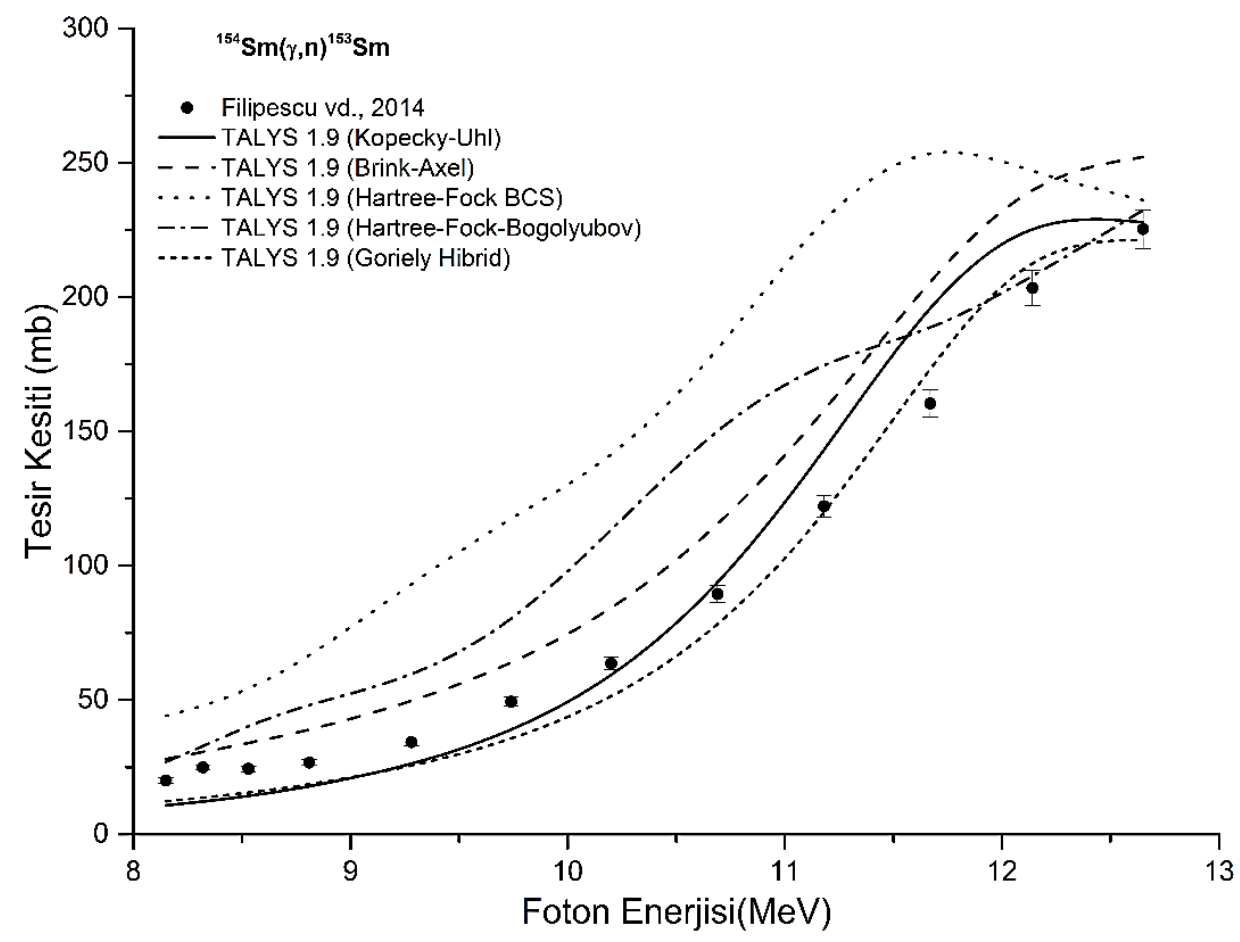

Şekil 4. ${ }^{154} \mathrm{Sm}(\gamma, \mathrm{n}){ }^{153} \mathrm{Sm}$ reaksiyonu için gama kuvvet fonksiyonları ile elde edilen teorik tesir kesiti hesaplamalarının deneysel veriler ile kıyaslanması

Sırasıyla Şekil 5-8'de ise ${ }^{148} \mathrm{Sm}(\gamma, 2 \mathrm{n}){ }^{146} \mathrm{Sm}$, fonksiyonlarının kullanılması sonucunda elde ${ }^{150} \mathrm{Sm}(\gamma, 2 \mathrm{n}){ }^{148} \mathrm{Sm}, \quad{ }^{152} \mathrm{Sm}(\gamma, 2 \mathrm{n}){ }^{150} \mathrm{Sm} \quad$ ve edilen sonuçlar ve bu sonuçların ${ }^{154} \mathrm{Sm}(\gamma, 2 \mathrm{n}){ }^{152} \mathrm{Sm}$ reaksiyonlarının tesir kesiti karşılaştııılması amacıyla kullanılan Carlos vd. hesaplamalarında gama kuvvet (1974)'ye ait deneysel veriler gösterilmiştir. Bu 
çalışmada incelenen $(\gamma, 2 \mathrm{n})$ reaksiyonlarında, edileceği hem Şekil 5-8'de gösterilen grafiksel deneysel veriler ile en uyumlu tesir kesiti karşılaştırmalardan hem de Tablo 1 ve Tablo hesaplamalarının Brink-Axel gama kuvvet 2'de verilmiş olan sayısal sonuçlardan fonksiyonu kullanılması durumunda elde anlaşılmıştır.

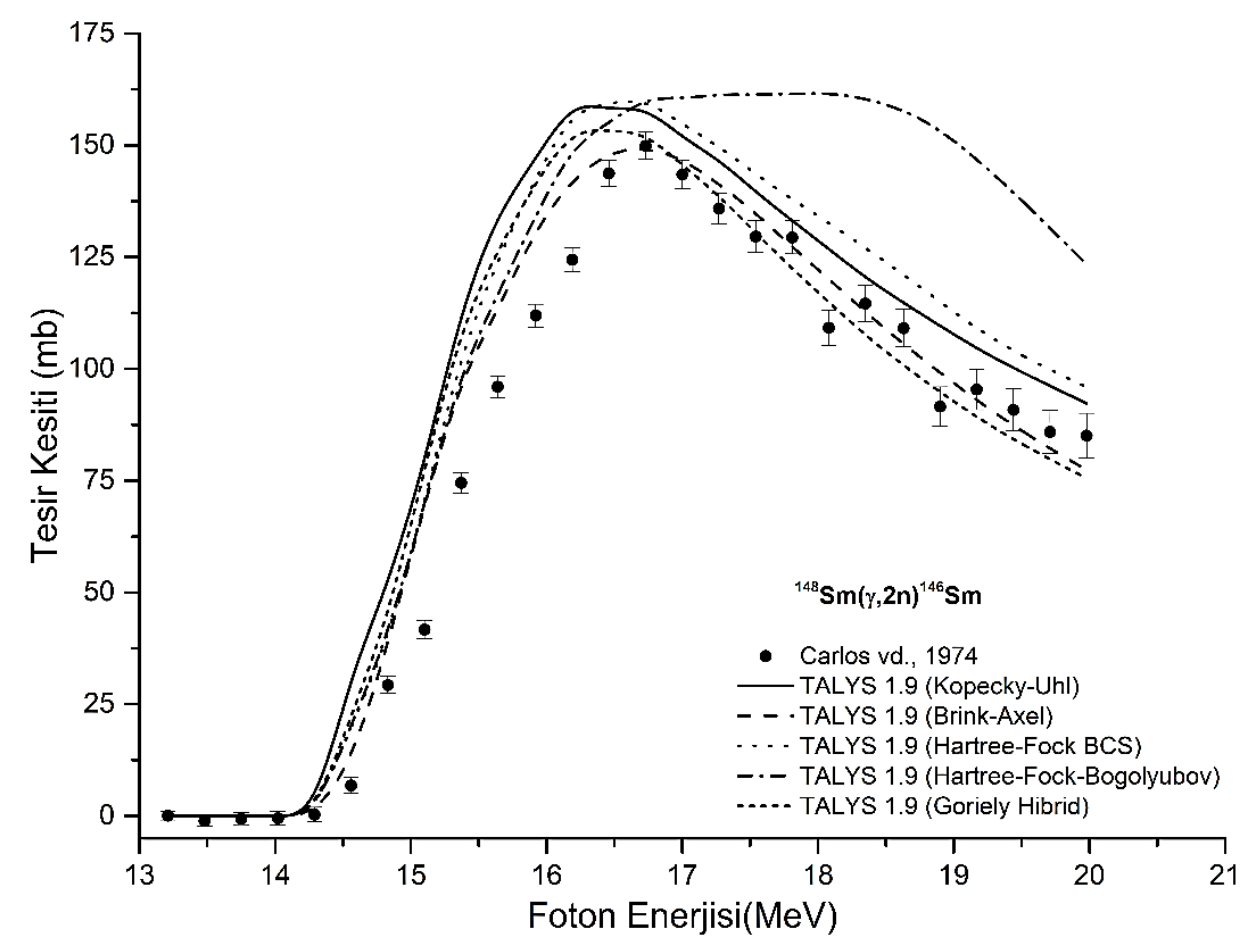

Şekil 5. ${ }^{148} \mathrm{Sm}(\gamma, 2 \mathrm{n}){ }^{146} \mathrm{Sm}$ reaksiyonu için gama kuvvet fonksiyonları ile elde edilen teorik tesir kesiti hesaplamalarının deneysel veriler ile kıyaslanması

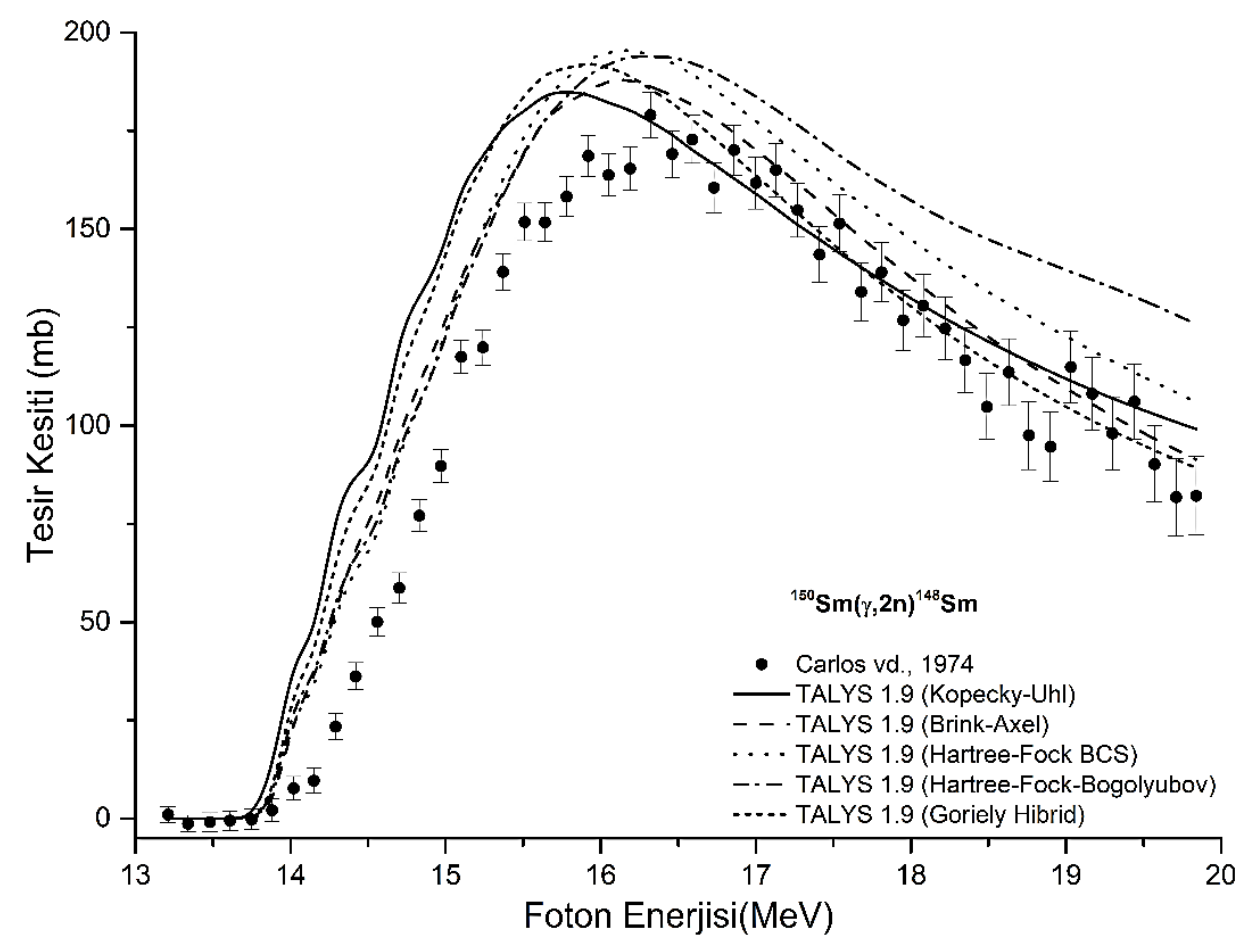

Şekil 6. ${ }^{150} \mathrm{Sm}(\gamma, 2 \mathrm{n}){ }^{148} \mathrm{Sm}$ reaksiyonu için gama kuvvet fonksiyonları ile elde edilen teorik tesir kesiti hesaplamalarının deneysel veriler ile kıyaslanması 


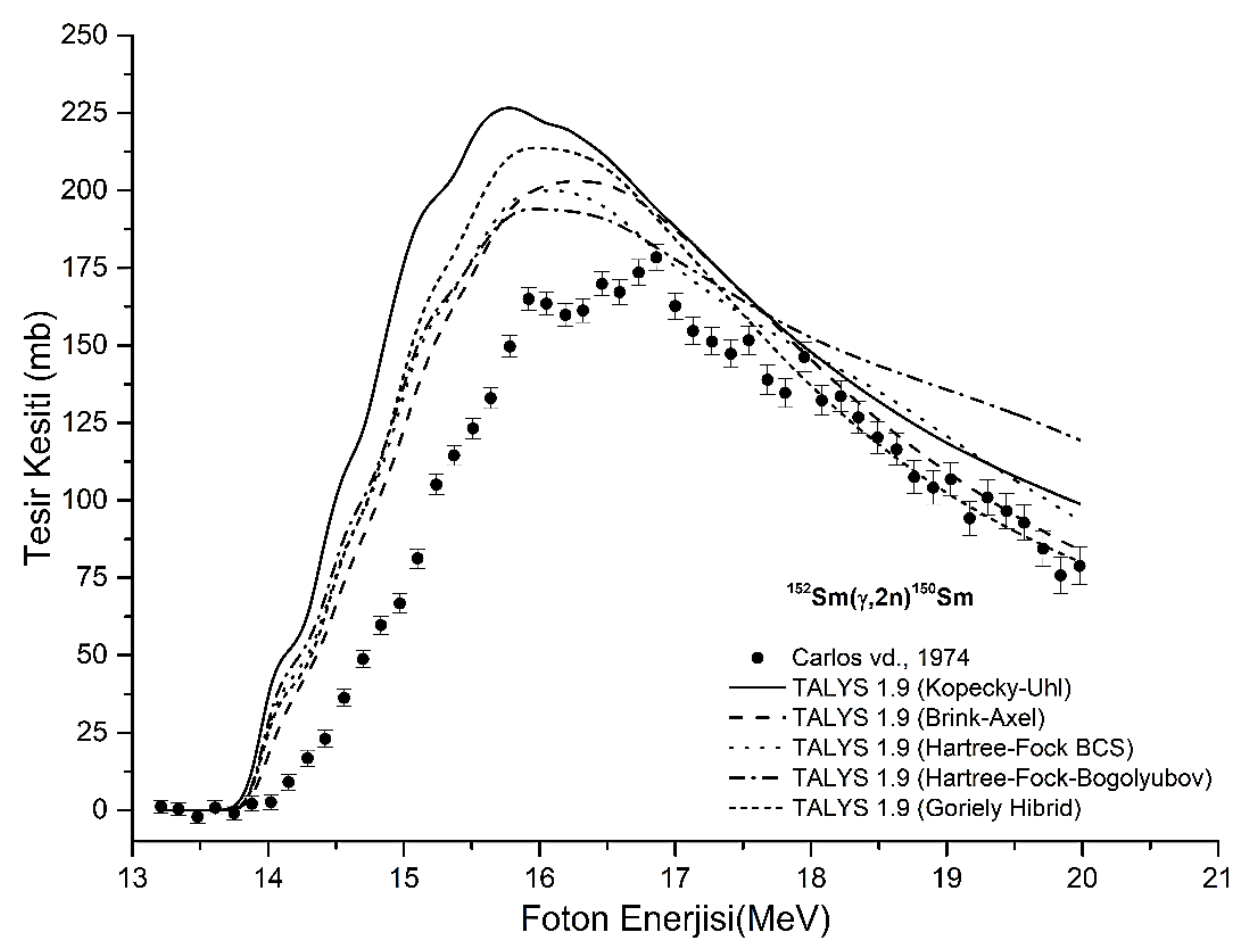

Şekil 7. ${ }^{152} \mathrm{Sm}(\gamma, 2 \mathrm{n}){ }^{150} \mathrm{Sm}$ reaksiyonu için gama kuvvet fonksiyonları ile elde edilen teorik tesir kesiti hesaplamalarının deneysel veriler ile kıyaslanması

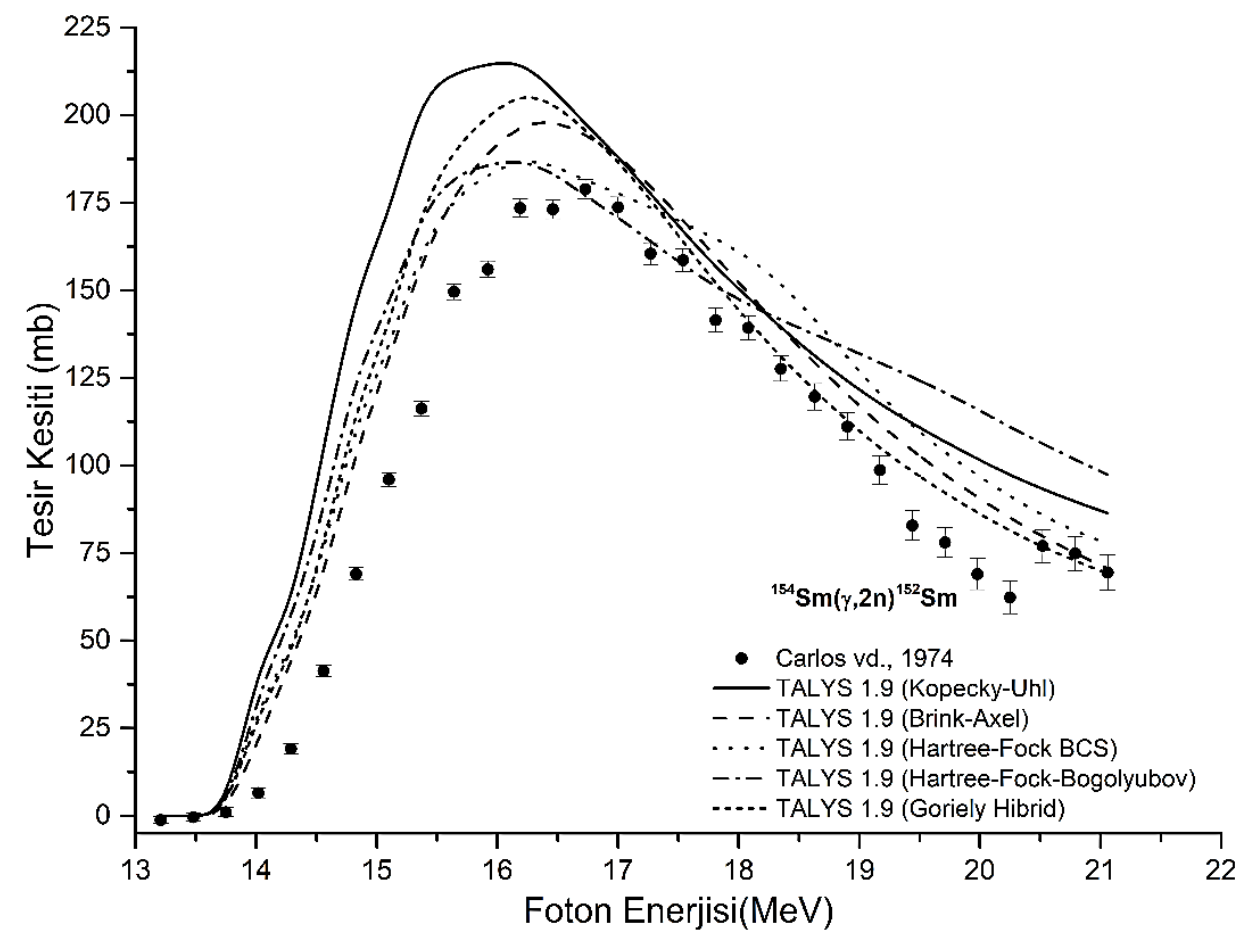

Şekil 8. ${ }^{154} \mathrm{Sm}(\gamma, 2 \mathrm{n}){ }^{152} \mathrm{Sm}$ reaksiyonu için gama kuvvet fonksiyonları ile elde edilen teorik tesir kesiti hesaplamalarının deneysel veriler ile kıyaslanması 
Tablo 1. Hesaplama sonuçlarının deneysel verilere göre bağıl varyans analizi

\begin{tabular}{|c|c|c|c|c|c|}
\hline Reaksiyon & $\begin{array}{c}\text { TALYS 1.9 } \\
\text { (Kopecky-Uhl) }\end{array}$ & $\begin{array}{c}\text { TALYS 1.9 } \\
\text { (Brink-Axel) }\end{array}$ & $\begin{array}{c}\text { TALYS 1.9 } \\
\text { (Hartree-Fock BCS) }\end{array}$ & $\begin{array}{c}\text { TALYS 1.9 } \\
\text { (Hartree-Fock- } \\
\text { Bogolyubov) }\end{array}$ & $\begin{array}{c}\text { TALYS 1.9 } \\
\text { (Goriely Hibrid) }\end{array}$ \\
\hline${ }^{148} \mathrm{Sm}(\gamma, \mathrm{n})^{147} \mathrm{Sm}$ & 0.328996028 & 0.363266767 & 0.885328236 & 0.340385398 & 0.228277828 \\
\hline$\left.{ }^{150} \mathrm{Sm}(\gamma, \mathrm{n})\right)^{149} \mathrm{Sm}$ & 0.251911422 & 0.467198197 & 0.925455275 & 0.332803435 & 0.18161949 \\
\hline${ }^{152} \mathrm{Sm}(\gamma, \mathrm{n}){ }^{151} \mathrm{Sm}$ & 0.286532919 & 0.215582148 & 0.883572777 & 0.29557413 & 0.306646978 \\
\hline$\left.{ }^{154} \mathrm{Sm}(\gamma, \mathrm{n})\right)^{153} \mathrm{Sm}$ & 0.241220459 & 0.303573599 & 0.98774326 & 0.462588823 & 0.223450186 \\
\hline${ }^{148} \mathrm{Sm}(\gamma, 2 \mathrm{n}){ }^{146} \mathrm{Sm}$ & 0.274247628 & 0.080037854 & 0.191731064 & 0.307176386 & 0.179615548 \\
\hline${ }^{150} \mathrm{Sm}(\gamma, 2 \mathrm{n}){ }^{148} \mathrm{Sm}$ & 0.406219592 & 0.196676492 & 0.237767965 & 0.310680935 & 0.280576033 \\
\hline${ }^{152} \mathrm{Sm}(\gamma, 2 \mathrm{n})^{150} \mathrm{Sm}$ & 0.925335481 & 0.45458653 & 0.612586192 & 0.707797576 & 0.587382221 \\
\hline${ }^{154} \mathrm{Sm}(\gamma, 2 \mathrm{n}){ }^{152} \mathrm{Sm}$ & 0.526921566 & 0.246699294 & 0.340160156 & 0.448430659 & 0.295978727 \\
\hline
\end{tabular}

Tablo 2. Hesaplama sonuçlarının deneysel verilere göre ortalama ağırlıklı sapma analizi

\begin{tabular}{|c|c|c|c|c|c|}
\hline Reaksiyon & $\begin{array}{c}\text { TALYS 1.9 } \\
\text { (Kopecky-Uhl) }\end{array}$ & $\begin{array}{c}\text { TALYS 1.9 } \\
\text { (Brink-Axel) }\end{array}$ & $\begin{array}{c}\text { TALYS 1.9 } \\
\text { (Hartree-Fock BCS) }\end{array}$ & $\begin{array}{c}\text { TALYS 1.9 } \\
\text { (Hartree-Fock- } \\
\text { Bogolyubov) }\end{array}$ & $\begin{array}{c}\text { TALYS 1.9 } \\
\text { (Goriely Hibrid) }\end{array}$ \\
\hline${ }^{148} \mathrm{Sm}(\gamma, \mathrm{n})^{147} \mathrm{Sm}$ & 7.134975815 & 9.281421823 & 22.46479901 & 11.46473798 & 6.258480909 \\
\hline${ }^{150} \mathrm{Sm}(\gamma, \mathrm{n}){ }^{149} \mathrm{Sm}$ & 5.530313936 & 10.95602446 & 18.55866826 & 9.336372608 & 4.353975158 \\
\hline${ }^{152} \mathrm{Sm}(\gamma, \mathrm{n}){ }^{151} \mathrm{Sm}$ & 4.916426751 & 4.76847897 & 13.71915999 & 5.182059418 & 4.769277949 \\
\hline$\left.{ }^{154} \mathrm{Sm}(\gamma, \mathrm{n})\right)^{153} \mathrm{Sm}$ & 6.965323499 & 8.08292691 & 27.8482682 & 13.90719674 & 6.370659365 \\
\hline${ }^{148} \mathrm{Sm}(\gamma, 2 \mathrm{n}){ }^{146} \mathrm{Sm}$ & 7.927083313 & 4.311067062 & 6.3544644 & 8.582680416 & 6.459924545 \\
\hline${ }^{150} \mathrm{Sm}(\gamma, 2 \mathrm{n})^{148} \mathrm{Sm}$ & 6.34202254 & 4.081508202 & 4.230214849 & 4.75240361 & 5.670822077 \\
\hline${ }^{152} \mathrm{Sm}(\gamma, 2 \mathrm{n})^{150} \mathrm{Sm}$ & 14.80711905 & 8.298962998 & 9.320381307 & 9.944037627 & 10.59282726 \\
\hline${ }^{154} \mathrm{Sm}(\gamma, 2 \mathrm{n})^{152} \mathrm{Sm}$ & 18.50730056 & 9.137384419 & 10.30332775 & 13.06582868 & 11.60226865 \\
\hline
\end{tabular}

\section{Sonuç ve Tartışma}

$\mathrm{Bu}$ çalışmada; birçok izotopu ve çeşitli kimyasal bileşikleri, endüstriyel ve medikal alanlarda başta olmak üzere farklı uygulamada kullanılan samaryumun ${ }^{148,150,152,154}$ Sm izotoplarında $(\gamma, n)$ ve $(\gamma, 2 n)$ reaksiyonlarının tesir kesiti hesaplamalarına gama kuvvet fonksiyonlarının etkilerinin araştırılması amaçlanmıştır. $\mathrm{Bu}$ amaç doğrultusunda, incelenen her reaksiyon için TALYS 1.9 kodunda mevcut olan KopeckyUhl genelleştirilmiş Lorentzian, Brink-Axel Lorentzian, Hartree-Fock BCS tablolar1, Hartree-Fock-Bogolyubov tabloları ve Goriely'nin hibrit model gama kuvvet fonksiyonları kullanılarak tesir kesiti hesaplamaları yapılmış ve elde edilen sonuçlar literatürde mevcut olan deneysel veriler ile kıyaslanmıştır. Hem grafiksel olarak hem de sayısal olarak yapilan karşılaştırmalar sonucunda elde edilen bulgular şu şekilde yorumlanabilir:
- Aynı reaksiyon ve enerjisi aralığında dahi, tesir kesiti hesaplamalarında farklı gama kuvvet fonksiyonlarının kullanılması durumunda hesaplama sonuçlarında farklılıklar oluşabilmektedir.

- Bu çalışmada incelenen tüm reaksiyonlarda, hesaplama sonuçlarında elde edilen tesir kesiti değerleri ile deneysel veriler arasında genel olarak benzer harmoni gözlenmiş olmasına rağmen tüm reaksiyonlar için deneysel veriler ile en uyumlu sonuçların elde edilmesinde tek bir model olduğu söylenemez.

$-{ }^{148} \mathrm{Sm}(\gamma, n){ }^{147} \mathrm{Sm},{ }^{150} \mathrm{Sm}(\gamma, \mathrm{n}){ }^{149} \mathrm{Sm}$ ve
${ }^{154} \mathrm{Sm}(\gamma, \mathrm{n}){ }^{153} \mathrm{Sm}$ reaksiyonlarında deneysel veriler ile en uyumlu sonuçlar incelenen tüm enerji aralığı düşünüldüğünde Goriely’nin hibrit model gama kuvvet fonksiyonları kullanılarak elde edilmişken diğer reaksiyonlarda incelenen tüm enerji aralıkları göz önüne alındığında deneysel veriler ile en uyumlu sonuçların Brink-Axel gama kuvvet fonksiyonunun kullanılması durumunda elde edildiği görülmüştür. 
- İncelenen samaryum izotoplarının $(\gamma, \mathrm{n})$, $(\gamma, 2 \mathrm{n})$ ve diğer Dev Dipol Rezonans (Giant Dipole Resonance, GDR) bölgesine etki eden reaksiyonların bir arada incelenmesi ile yeni GDR parametrelerinin elde edilmesi ve bunların TALYS koduna eklenerek tesir kesiti hesaplamalarındaki etkilerinin incelenmesi mümkündür.

- Farklı reaksiyonlar için en uyumlu gama kuvvet fonksiyonlarının bulunması, tesir kesiti hesaplamalarına etki eden diğer parametrelerin incelenmesinde zaman kazanılmasina ve diğer parametreler ile gama kuvvet fonksiyonları arasındaki ilişkilerin de incelenmesine olanak sağlayacaktır.

- Açıklanan bu gerekçeler sayesinde, teorik modellerin iyileştirilmesine geliştirilmesine katkı sağlanması da mümkündür.

\section{References}

Axel, P., 1962. "Electric dipole ground-state transition width strength function and 7-MeV photon interactions". Physical Review, 126, 671.

Ay, K. O., 2019. “'149,151 Nd Çekirdeklerinin Nükleer Düzey Yoğunluklarının ve Gama Kuvvet Fonksiyonlarının İncelenmesi”, Eskişehir Osmangazi Üniversitesi, Fen Bilimleri Enstitüsü, Eskişehir, Doktora Tezi, $98 \mathrm{~s}$.

Aydin, A., Pekdogan, H., Kaplan, A., Sarpün, İ. H., Tel, E., Demir, B., 2015. "Comparison of Level Density Models for the ${ }^{60,61,62,64} \mathrm{Ni}(\mathrm{p}, \mathrm{n})$ Reactions of Structural Fusion Material Nickel from Threshold to $30 \mathrm{MeV}$ '. Journal of Fusion Energy, 34(5), 1105-1108.

Aydın, A., Yalım, H. A., Tel, E., Şarer, B., Ünal, R., Sarpün, İ. H., Kaplan, A., Dağ, M., 2009. "Level density parameter dependence of the fission cross sections of some subactinide nuclei induced by protons with the incident energy up to $250 \mathrm{MeV}$ ". Annals of Nuclear Energy, 36(9), 1307-1312.

Baernighausen, H., Haschke, J. M., 1978. "Compositions and crystal structures of the intermediate phases in the samarium-bromine system". Inorganic Chemistry, 17, 18-21.
Bartholomew, G. A., Earle, E. D., Ferguson, A. J., Knowles, J. W., Lone, M. A., 1973. "Gamma-Ray Strength Functions", Advances in Nuclear Physics, 7, Springer, Boston, USA, 229-324.

Becquerel, H., 1896. "Sur les radiations émises par phosphorescence". Comptes rendus de l'Académie des Sciences, 122, 420421.

Brink, D. M., 1957. "Individual particle and collective aspects of the nuclear photoeffect". Nuclear Physics, 4, 215.

Canbula, B., 2017. "Bazı tellür izotoplarının nötron yakalama tesir kesiti analizi". Celal Bayar Üniversitesi Fen Bilimleri Dergisi, 13(2), 445-455.

Capote, R., Herman, M., Obložinský, P., Young, P. G., Goriely, S., Belgya, T., Ignatyuk, A. V., Koning, A. J., Hilaire, S., Plujko, V. A., Avrigeanu, M., Bersillon, O., Chadwick, M. B., Fukahori, T., Ge, Z., Han, Y., Kailas, S., Kopecky, J., Maslov, V. M., Reffo, G., Sin, M., Soukhovitskii, E. S., Talou, P., 2009. "RIPL - reference input parameter library for calculation of nuclear reactions and nuclear data evaluations". Nuclear Data Sheets, 110, 3107.

Carlos, P., Beil, H., Bergère, R., Leprêtre, A., Miniac, A. De., Veyssière, A., 1974. "The giant dipole resonance in the transition region of the samarium isotopes". Nuclear Physics A, 225(1), 171-188.

Chu, S., 2011. "U.S. Department of Energy Critical Materials Strategy", DIANE Publishing.

Coursey, J. S., Schwab, D. J., Tsai, J. J., Dragoset, R. A., 2015. "Atomic Weights and Isotopic Compositions (version 4.1)", National Institute of Standards and Technology, https://www.nist.gov/pml/atomic-weights-andisotopic-compositions-relative-atomic-masses, Erişim Tarihi: 25.03.2020

Demir, B., Kaplan, A., Çapalı, V., Sarpün, İ. H., Aydın, A., Tel, E., 2015. "Production cross-section calculations of medical ${ }^{32} \mathrm{P}$, ${ }^{11} 7 \mathrm{Sn},{ }^{153} \mathrm{Sm}$ and ${ }^{186,188} \mathrm{Re}$ radionuclides used in bone pain palliation treatment", Kerntechnik, 80(1), 58-65.

Emsley, J., 2011. "Nature's Building Blocks: An A-Z Guide to the Elements", Oxford University Press, New York, USA, 2nd Edition, 2011, $720 \mathrm{~s}$. 
Evans, W. J., Hughes, L. A., Hanusa, T. P., 1986. "Synthesis and x-ray crystal structure of bis(pentamethylcyclopentadienyl) complexes of samarium and europium: (C5Me5)2Sm and (C5Me5)2Eu". Organometallics, 5(7), 12851291.

Filipescu, D. M., Gheorghe, I., Utsunomiya, H., Goriely, S., Renstrøm, T., Nyhus, H. T., Tesileanu, O., Glodariu, T., Shima, T., Takahisa, K., Miyamoto, S., Lui, Y. W., Hilaire, S., Péru, S., Martini, M., Koning, A. J., 2014. "Photoneutron cross sections for samarium isotopes: Toward a unified understanding of $(\gamma, n)$ and $(n, \gamma)$ reactions in the rare earth region". Physical Review C, 90, 064616.

Hara, K. Y., Harada, H., Kitatani, F., Goko, S., Hohara, S., Kaihori, T., Makinaga, A., Utsunomiya, H., Toyokawa, H., Yamada, K., 2007. "Measurements of the ${ }^{152} \operatorname{Srn}(\gamma, n)$ Cross Section with Laser-Compton Scattering $\gamma$ Rays and the Photon Difference Method". Journal of Nuclear Science and Technology, 44(7), 938945.

Haynes, W. M., 2014. "CRC Handbook of Chemistry and Physics, 95th Edition", CRC Press, Hoboken, New Jersey, USA, 2704 s.

Holmes, R. A., 1992. "[ ${ }^{153}$ Sm]EDTMP: A potential therapy for bone cancer pain", Seminars in Nuclear Medicine, 22(1), 41-45.

Jayaraman, A., Narayanamurti, V., Bucher, E., Maines, R., 1970. "Continuous and Discontinuous Semiconductor-Metal Transition in Samarium Monochalcogenides Under Pressure". Physical Review Letters, 25 (20), 1430.

Kaplan, A., Özdoğan H., Aydin, A., Tel, E., 2014. "Photo-Neutron Cross-Section Calculations of ${ }^{142,143,144,145,146,150} \mathrm{Nd}$ Rare-Earth Isotopes for (g,n) Reaction". Physics of Atomic Nuclei, 77(11), 1371-1377.

Kaplan, A., Özdoğan, H., Aydın, A., Tel, E., 2013. "Deuteron-Induced Cross Section Calculations of Some Structural Fusion Materials". Journal of Fusion Energy, 32(1), 97-102.

Kara, A., Yiğit, M., Korkut, T., Tel, E., 2015. "Cross Section Calculations of Neutron Induced Reactions on $124,126,128,134,136 \mathrm{Xe}$ ". Journal of Fusion Energy, 34, 882-886.

Kavun, Y., Tel, E., Şahan, M., Salan, A., 2019. "Calculation of Production Reaction Cross
Section of Some Radiopharmaceuticals Used in Nuclear Medicine by New Density Dependent Parameters". Süleyman Demirel Üniversitesi Fen Edebiyat Fakültesi Fen Dergisi, 14(1), 5761.

Kolesnikov-Gauthier, H., Lemoine, N., TreschBruneel, E., Olivier, A., Oudoux, A., Penel, N., 2018. "Efficacy and safety of ${ }^{153}$ Sm-EDTMP as treatment of painful bone metastasis: a large single-center study", Supportive Care in Cancer, 26, 751-758.

Koning, A., Hilaire, S., Goriely, S., 2017. TALYS-1.9 A Nuclear Reaction Program, User Manual, 1st ed. 21 December 2017.

Kopecky, J., Uhl, M., 1990. "Test of gammaray strength functions in nuclear reaction model calculations". Physical Review C, 41, 1941.

Kurenkov, N., Lunev, V., Shubin, Y., 1999. "Evaluation of calculation methods for excitation functions for production of radioisotopes of iodine, thallium and other elements". Applied Radiation and Isotopes, 50, 541-549.

Leger, J., Yacoubi, N., Loriers, J., 1981. "Synthesis of rare earth monoxides". Journal of Solid State Chemistry, 36(3), 261.

Lone, M. A., 1979. "Photon Strength Functions", Neutron Capture Gamma-Ray Spectroscopy, Springer, Boston, USA, 161180.

Martin, B. R., 2006. "Nuclear and Particle Physics". John Wiley \& Sons, Ltd.

Martin, D., 2012. "Do we understand gamma strength functions? The case of 96Mo", Institut für Kernphysik Technische Universitat Darmstadt.

http://tid.uio.no/workshop2013/talks/Oslo13_s 413_Martin.pdf, Erişim Tarihi: 25.03.2020

Özdoğan, H., 2018. “181 $\mathrm{Ta}(\alpha, \mathrm{xn})$ Reaksiyonu Tesir Kesitlerinin İncelenmesi”. Süleyman Demirel Üniversitesi Fen Edebiyat Fakültesi Fen Dergisi, 13(2), 54-66.

Özdoğan, H., Şekerci, M., Kaplan, A., 2019. "Investigation of gamma strength functions and level density models effects on photon induced reaction cross-section calculations for the fusion structural materials ${ }^{46,50} \mathrm{Ti},{ }^{51} \mathrm{~V},{ }^{58} \mathrm{Ni}$ and ${ }^{63} \mathrm{Cu}$, Applied Radiation and Isotopes, 143, 610. 
Özdoğan, H., Şekerci, M., Sarpün, İ. H., Yiğit, M., Bostan, S. N., 2019. "Study on cross

Kaplan, A., 2018. "Investigation of level density parameter effects on $(\mathrm{p}, \mathrm{n})$ and $(\mathrm{p}, 2 \mathrm{n})$ reaction cross-sections for the fusion structural materials ${ }^{48} \mathrm{Ti},{ }^{63} \mathrm{Cu}$ and ${ }^{90} \mathrm{Zr} "$. Applied Radiation and Isotopes, 140, 29-34.

Özgür, M., 2017. "Neodimyum-144,145 Çekilrdeklerinin Durum Yoğunlukları ve Gama Kuvvet Fonksiyonları", Eskişehir Osmangazi Üniversitesi, Fen Bilimleri Enstitüsü, Yüksel Lisans Tezi, Eskişehir, $65 \mathrm{~s}$.

Sarpün, İ. H., Özdoğan, H., Taşdöven, K., Yalim, H. A., Kaplan, A., 2019. "Theoretical photoneutron cross-section calculations on Osmium isotopes by Talys and Empire codes". Modern Physics Letters A, 34(26), 1950210.

Şekerci M., Özdoğan H., Kaplan A., 2019. "Investigation on the Different Production Routes of ${ }^{67} \mathrm{Ga}$ Radioisotope by Using Different Level Density Models". Moscow University Physics Bulletin, 74, 277-281.

Şekerci M., Özdoğan H., Kaplan A., 2020. "An investigation of effects of level density models and gamma ray strength functions on cross-section calculations for the production of ${ }^{90} \mathrm{Y},{ }^{153} \mathrm{Sm},{ }^{169} \mathrm{Er},{ }^{177} \mathrm{Lu}$ and ${ }^{186} \mathrm{Re}$ therapeutic radioisotopes via $(\mathrm{n}, \mathrm{g})$ reactions", Radiochimica Acta, 108, 11-17.

Spedding, F. H., Gschneidner, K., Daane, A. H., 1958. "The Crystal Structures of Some of the Rare Earth Carbides". Journal of the American Chemical Society, 80(17), 44994503. section calculations for (n,p) nuclear reactions of cadmium isotopes", Applied Radiation and Isotopes, 154, 108868.

Yiğit, M., Tel, E., Sarpün, İ. H., 2016. "Excitation function calculations for $\alpha+{ }^{93} \mathrm{Nb}$ nuclear reactions". Nuclear Instruments and Methods in Physics Research Section B: Beam Interactions with Materials and Atoms, 385, 59-64.

Yiğit, M., Tel, E., Tanır, G., 2013. "Calculations of Proton Emission Cross Sections in Deuteron Induced Reactions of Some Fusion Structural Materials". Journal of Fusion Energy, 32, 317-321.

Zerkin, V. V., Pritychenko, B., 2018. "The Experimental Nuclear Reaction Data (EXFOR): Extended Computer Database and Web Retrieval System". Nuclear Instruments and Methods in Physics Research Section A: Accelerators, Spectrometers, Detectors and Associated Equipment, 888, 31-43. 